\title{
Communication Conflictuelle De Fin De Regne En RDC.
}

\section{Vincent Mukwege Buhendwa}

De la fermeture de la Maison Schengen de Kinshasa à l'investiture de Fatshi.

\section{Resume}

La transition politique survenue en République Démocratique du Congo (RDC), en janvier 2019, fut le résultat- inespéré- de nombreux affrontements. Dans cette bataille rangée, quatre types de belligérants, armés jusqu'aux dents, repartis en «variables », étaient déterminés à marquer, de leurs empreintes, ce tournant politique, unique en son genre. D'une part, les « Variables Internes Favorables au Glissement » ont été appuyées, de l'extérieur, par les « Variables Externes Favorables au Glissement ». D'autre part, ce duo du statu quo se frottait constamment à la résistance farouche des «Variables Internes Défavorables au Glissement», appuyées, de l'extérieur, par les «Variables Externes Défavorables au Glissement ». Les résultats de cette étude retracent un essai de chronique de ces affrontements où les locuteurs/acteurs ont joint l'acte à la parole, de la fermeture de la Maison Schengen de Kinshasa à l'investiture de Félix-Antoine Tshisekedi Tshilombo (Fatshi), dit «élu Président » de la République. Le point d'orgue de ces affrontements marque plutôt le dénouement d'une conflictualité qui aurait pu dégénérer en une crise post-électorale aux dimensions incommensurables, mais qui plutôt, par un tour de manœuvre, a privilégié une vérité socialement (re)construite, au regard des réalités et impératifs du moment, par ailleurs inhérents à la realpolitik.

\section{Mots-clés : affrontement-variables-internes-externes-favorables-défavorables-pouvoir.}

\section{Introduction}

La conflictualité politique congolaise a cristallisé des peurs et des frustrations, de nature à inhiber toute tentative de communication sur un passé récent de fin de règne. Un contexte pareil paralyse les efforts individuels et collectifs de quête de sens et empêche des citoyens éclairés de s'approprier les faits historiques de leur pays. Aussi cette étude vise-t-elle à surmonter cette peur- combien paralysante- afin de reconstituer, autant que faire ce peut, quelques scenarii et leurs paysages ; replacer les mots sur les faits, et, par-ricochet, (re)donner sens aux événements sociopolitiques récents, sans jamais prétendre infléchir le cours de ces derniers. Cela paraît d'autant plus pertinent que l'arène politique, d'où surgissent des discours discordants, est aussi-à n'en point douter- celle où les acteurs, mis en scène, exercent la manipulation les uns sur les autres. Et, traitant de la stratégie manipulatoire, M. Beauvallet observe qu' «Il y a deux méthodes pour faire baisser la fièvre : se soigner ou trafiquer le thermomètre. La deuxième suppose que vous ayez la maîtrise du thermomètre. Et c'est souvent le cas $\gg 1$.

Cette chronique fait suite à notre publication du 27 juin $2020^{2}$ qui a traité de l' «Effet contagieux de l'idéologie contestataire. De la chute du Mur de Berlin à l'Accord de la Saint-Sylvestre » en RDC. Aussi rebondit-elle sur quelques confrontations et affrontements qui ont caractérisé la communication conflictuelle de fin de règne en RDC, de la fermeture de la Maison Schengen de Kinshasa à la passation dite « civilisée » du pouvoir entre Fatshi et Joseph Kabila Kabange (JKK). Elle récence 122 faits et gestes qui tous, à quelque exception près, sont inscrits au registre des variables internes et externes, pro ou anti-glissement.

Les résultats de cette étude pointent du doigt quelques facteurs-clés, déclencheurs de l'alternance politique congolaise, dont (1) la fermeture de la Maison Schengen de Kinshasa ; (2)l'annulation de la visite papale; (3)les actions musclées du $\mathrm{CLC}^{3}$ et de la $\mathrm{CENCO}^{4}$; (4) la coalition des Eglises catholiques et

\footnotetext{
${ }^{1}$ M.BEAUVALLET, Les stratégies absurdes. Comment faire pire en croyant faire mieux. Ed. du Seuil, Paris,2009, p.10.

${ }^{2} \mathrm{https}$ //www.thinkingafrica.org/V2/chronique-de-la-communication-conflictuelle-de-fin-de-regne-en-rdc/ (7 mars 2021)
} 
protestantes contre le régime de JKK ; (5) l'acharnement de Washington sur Kinshasa ; (6) l'assassinat des deux experts de l'ONU ${ }^{5}$ - Zaida Catalan \& Michael Sharp sur le sol congolais ; (7) le rêve (chimérique) d'une transition sans Kabila; (8) la prise de parole de JKK qui sort de son mutisme ; (9) le tournant irréversible de la machine électorale ; (10)le feuilleton Moïse Katumbi, la Machine à Voter( la MAV)-dite machine à «fauter », machine à « voler »- et le renoncement de JKK au $3^{\mathrm{e}}$ mandat ; (11) la guéguerre des Mouvements citoyens-LUCHA ${ }^{6}$, Filimbi, Compte à rebours et consorts- contre le régime de JKK, laquelle guéguerre fut récupérée-et détournée- par une poignée d'opposants politiques opportunistes ; (12) la désignation du dauphin par JKK; (13) le règlement des comptes JKK-Moïse Katumbi, le black-out médiatique au lendemain d'une élection truquée; (14) les tractations de JKK de dernière minute et la passation dite « civilisée » du pouvoir, autant de questions couvertes d'un silence de cimetière qui, pourtant, n'a pas freiné la marche de la RDC et des Congolais vers leur destin qu'ils tiennent, à tout prix, à transformer en destinée.

\section{II.Question De Recherche, Hypotheses Et Methode}

Cette étude est partie d'une interrogation : "les 'belligérants' récemment observés sur la scène politique congolaise sont-ils les seuls et réels acteurs à en avoir déterminé, les uns l'épuisement, l'effondrement du défunt régime ; et les autres, les efforts d'autoconservation? ». Subsidiairement à cette question, « quels sont les faits et gestes saillants, teintés de communication conflictuelle observés, de la fermeture de la Maison Schengen de Kinshasa à l'investiture de Fatshi ? ».

Le rapprochement des variables internes, favorables et défavorables au glissement du calendrier électoral, relève de la realpolitik mais davantage du dépassement des contradictions internes, en vue d'une solution durable à la crise politique congolaise. Le déséquilibre des forces internes entre anti-et proglissement, qui a caractérisé les affrontements ayant mené à l'alternance politique, a largement bénéficié d'une pression externe, fruit de l'ingérence dans les affaires intérieures de l'Etat congolais. Telles sont les hypothèses de la présente étude. L'approche méthodologique de la recherche procède par la construction d'un corpus constitué de matériaux issus des messages, faits et gestes qui ont, de manière significative, marqué les deux années pré-électorales (2017 et 2018). Ces matériaux sont présentés et analysés sous différentes grilles de lecture empruntées à l'idéologie contestataire, la communication conflictuelle, la pragmatique de la communication et la dialectique qui, toutes, fécondent l'avènement d'une réalité ou vérité socialement (re)construite. Cet arsenal méthodologique est consolidé par le genre dramatique où les personnages parlent directement en leur nom et représentent une action ${ }^{7}$.

\section{Les Gouttes D'eau Qui Ont Fait Deborder Le Vase \\ III.1. Fermeture de la Maison Schengen, annulation de la visite papale...}

1. Fin janv.2017, Kinshasa ferme la Maison Schengen à Kinshasa, gérée par la Belgique, au compte de 18 Etats de l'UE $^{8}$ pour la délivrance des visas aux voyageurs en destination de l'Europe. Décision en guise de « représailles » à la celle de Bruxelles de priver le régime de JKK d'une cagnotte de 25 millions d'euros et qui seront désormais remis aux acteurs humanitaires ou de développement œuvrant en RDC. Privés de cette manne financière, les Kabilistes, désabusés, fulminent.

2. L'Accord de la Saint-Sylvestre difficilement acquis sous la médiation de la $\mathrm{CENCO}^{9}$ n'ayant pas été appliqué, le 14 mars 2017, le Pape François annonce qu'il annule sa visite en RDC, prévue entre juillet et août 2017. Sous un ton sec, le Saint Père s'indigne : "Il était prévu de me rendre aux deux Congo, mais

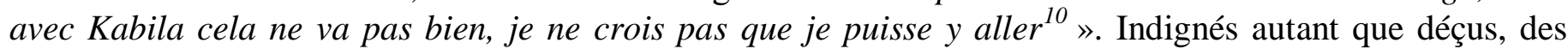
Congolais s'en prennent au régime Kabila, à l'origine de cet incident diplomatique. Réponse du berger à la bergère, Lambert Mende, gardien du temple kabiliste, Ministre de la Communication et Médias, monte au créneau et dénonce l'ingérence du Vatican dans les affaires intérieures de Kinshasa :

\footnotetext{
${ }^{5}$ Organisation des Nations Unies.

${ }^{6}$ Lutte pour le Changement-Mouvement Citoyen.

${ }^{7}$ J.D.MALLET, La tragédie et la comédie, Ed.Hatier, Saint-Amand, 2001, p.8.

${ }^{8}$ Union Européenne.

${ }^{9}$ Conférence Episcopale Nationale du Congo.

${ }^{10} \mathrm{http}: / /$ www.rfi.fr/afrique/20170314-le-pape-francois-annule-projet-visite-rdc (2 sept19)
} 
3. « la RDC n'est pas le seul pays sur la liste des pays que le Pape ne visitera pas; la Russie, le CongoBrazza et l'Ukraine y figurent également»; «Ce sont les autorités congolaises qui ont estimé que la situation sécuritaire ne se prête pas tellement pour que cette visite ait lieu... Le Président de la République a estimé qu'il faut attendre que la situation puisse évoluer». Lambert Mende n'est pas d'accord que l'annulation de la visite papale soit conséquente à la non-application de l'Accord de la Saint-Sylvestre: "Quand le Pape est allé en Syrie, y avait-il un accord qui a eu lieu ? Quand le Pape s'est rendu en Centrafrique, y avait-il un accord qui a eu lieu? C'est ridicule de lier la situation du pays avec la visite du Pape. On a besoin du Pape là où il y a des problèmes précisément ${ }^{11}$. Ainsi, tout en disant le contraire, Lambert Mende semble donner raison à ses détracteurs. A-t-il dit vrai ? A-t-il menti ? Les faits sont têtus !

4. Mgr Marcel Utembi, Président de la CENCO: «Nous sommes logiques et cohérents. L'accord souffre de sa mise en æuvre intégrale. Nous ne pouvons pas piloter un troisième dialogue, alors que la mise en æuvre $d u$ deuxième dialogue ne s'est pas réalisée intégralement ${ }^{12}$. Soutenus par l'opposition politique et la Société civile congolaise, les prélats congolais usent de la diplomatie de couloir pour pousser le régime dans la bonne direction. L'Abbé Donatien Nshole, Secrétaire Général et Porte-Parole de la CENCO, déclare: «Les évêques attendent de la Communauté internationale un appui pour la tenue des élections dans le meilleur délai $»^{13}$

5. Mai 2017, les Evêques de l'Assemblée Episcopale Provinciale de Bukavu (ASSEPB) ${ }^{14}$, dressent un état de lieu de la situation sociopolitique congolaise : «la classe politique a failli ». Ils exhortent les Congolais: «Faisons de la non-violence active notre style de vie ${ }^{15}{ }^{15}$ «L déficit de l'autorité de l'État est un constat établi depuis des décennies et qui va croissant : le pays est mal gouverné. (...) Le pays s'en trouve encore plus fragile que jamais. Sur le plan politique, il est exposé à tous les vents »16.

6. Le 3 juin 2017, JKK au journal allemand «Der Spiegel». Au sujet de la promesse qu'il aurait faite d'organiser les élections en 2017 : «Je n'ai rien promis du tout. Je souhaite organiser des élections aussi vite que possible »; "Nous voulons des élections parfaites, pas seulement des élections ». Quant à son éventuel $3^{\mathrm{e}}$ mandat, il réagit : "Cela dépend de ce qu'on entend vraiment par $3^{e}$ mandat»; "Nous $n$ 'avons en tout cas pas l'intention de porter atteinte à la Constitution. Et comment pourrais-je avoir un $3^{e}$ mandat sans porter atteinte à la Constitution ? » ${ }^{17}$

7. Le 12 nov.2017, Laurent Cardinal Monsengwo s'adresse à un groupe d'artistes de Kinshasa et les appelle à l'action: "Chacun de vous doit bien utiliser son talent au profit de la nation. C'est à partir de vos talents reçus du Créateur que chacun va concevoir des auvres qui forment et transforment l'Homme. La vraie transformation est celle qui respecte la nature» ${ }^{18}$.

8. JKK à l'Assemblée Générale de l'ONU où il prononce son discours le 23 sep.2017 à New York. Lambert Mende en apprécie la prestation et parle d'un " discours responsable ». Ses détracteurs contestent le discours d'un orateur «face à une communauté internationale qui souhaite son départ»; pour prononcer «un discours pour rien», «un discours vide dans une salle vide »; un discours "devant une Assemblée Générale de l'ONU aux rangs très clairsemés»; "Kabila à l'ONU : la preuve qu'il ne veut pas partir!»; «J'y suis et j'y reste ${ }^{19}$. Le discours de JKK a suscité un métadiscours qui est l'œuvre des partisans de l'anti-glissement. Ce métadiscours est polémique et traduit les divergences d'opinions entre le gouvernement et l'opposition. Là transparait la critique de l'adversaire par des procédés de la polémique, notamment l'accusation d'incompétence, de tromperie ${ }^{20}$.

\footnotetext{
${ }^{11}$ https://www.voaafrique.com/a/la-visite-du-pape-annulee-plutot-par-kinshasa-selon-mende/3767411.html (2sept19)

${ }^{12}$ https://www.lepoint.fr/afrique/rd-congo-la-situation-preoccupe-vraiment-les-eveques-catholiques-27-09-2017-2160309_3826.php (19 aout19)

${ }^{13}$ Ibid.

${ }^{14}$ Regroupant les diocèses de Bukavu (Archidiocèse), Goma, Beni-Butembo, Kindu, Kasongo et Uvira.

${ }^{15}$ La Croix du 2 juin 2017.

${ }^{16} \mathrm{Ibid}$.

${ }^{17}$ https://www.jeuneafrique.com/444942/politique/kabila-a-der-spiegel-pourrais-troisieme-mandat-porter-atteinte-a-constitution/ (2Sept19).

${ }^{18}$ https://www.francetvinfo.fr/monde/afrique/politique-africaine/rdc-laurent-monsengwo-l-archeveque-qui-veut-degager-le-mediocre-kabila_3055523.html (4aout19).

${ }^{19}$ https://www.lepoint.fr/afrique/rd-congo-kabila-a-1-onu-quelles-lecons-en-tirer-25-09-2017-2159780_3826.php\# (15juillet19)

${ }_{20}$ M.SANI, Communication conflictuelle, dans Essais $n^{\circ} 3-03$ (2010), p.76.
} 
9. A la tête d'une délégation en tournée en Europe, le 27 sept.2017, Mgr Marcel Utembi crie au loup : «l'heure est grave en RDC, il faut agir maintenant, avant qu'il ne soit trop tard »; alors que son confrère Mgr Fidèle Nsielele, Evêque de Kisantu et président de la Commission Episcopale Justice et Paix, rejette en bloc l'idée d'un troisième dialogue : "Pour la CENCO, un troisième dialogue ne servirait à rien. C'est possible de sortir de cette crise sans un autre dialogue. ${ }^{21}$.

10. Un groupe du CIAM $^{22}$ répond à l'appel de l'Eglise catholique et lance trois conférences-débats à Kinshasa. La première s'est tenue le 27 oct. 2017 à la Paroisse Saint Joseph de Matonge sur le thème « Décembre 2017, enjeux et défis ».Nous n'avons pas eu accès aux données du deuxième débat.

11. Le troisième débat, organisé à Ndjili, sert de tremplin au CLC qui lance un communiqué, appelant à la manifestation du 31 décembre 2017. Aussi faut-il admettre que les initiatives du CLC sont appuyées, sinon portées et financées par la CENCO, dirigée par Mgr Marcel Utembi, Mgr Fridolin Ambongo et Monsieur l'Abbé Donatien Nshole qui activement travaillent aux côtés de l'Archevêque de Kinshasa, Laurent Cardinal Monsengwo, anti-Kabila avéré !

\section{III.2. L'acharnement de l'Oncle Sam sur Kinshasa}

12. Baudouin Amba Wetshi, journaliste congolais, en appelle aux sanctions américaines contre le régime : "Vous savez, Mobutu était aussi de marbre, à l'époque où on l'avait privé de visa pour voyager aux USA. Mais, il en souffrait. Moi, je crois que le département (américain) du Trésor devrait prendre des sanctions identiques»; "On a touché l'entourage, mais jusqu'ici, Kabila et sa famille ne sont pas touchés. En tout cas, il faut que ça soit accompagné, comme on dit, par la carotte et le bâton. C'est la manière pour Kabila de comprendre ${ }^{23}$.

13. Cette option chère à Herman Cohen est soutenue par un Professeur d'Etudes Africaines de l'Université Johns Hopkins à Washington: «Les USA, pendant l'administration de Obama, ont déjà appliqué des sanctions contre de hautes personnalités, autour de Kabila, pour les pousser dans la bonne direction. S'ils ne voient pas un bon calendrier, avec une élection en 2018, ils peuvent étendre ces sanctions. Et aussi, ils n'ont jamais sanctionné la famille Kabila. Ça peut marcher, surtout si les Européens, Français et Belges notamment, sont avec les Américains ${ }^{24}$.

14. L'assassinat de deux experts de l'ONU, Zaida Catalan (Chillo-Suédoise) et Michael Sharp (Américain), le 12 mars 2017, ternit davantage l'image du régime de Kinshasa et sert de prétexte à Washington pour faire pression sur JKK. Entre-temps, l'investiture du Gouvernement Bruno Tshibala, dissident de l'UDPS ${ }^{25}$, par le Parlement, a lieu le 16 mai 2017.

15. La nuit du 17 mai 2017 intervient l'évasion de la prison de Makala de Kinshasa. Plus de 4600 détenus évadés à la faveur de l'intervention des combattants du chef spirituel de Bundi dia Kongo(BDK), venus massivement libérer leur «autorité morale », Ne Muanda Nsemi. Ce dernier et ses adeptes sont comptés parmi les évadés.

16. Au terme de son face à face avec le Président JKK à Kinshasa, le 27 oct.2017, l'Ambassadeur des EtatsUnis à l'ONU, Nikki Haley, donne l'essentiel de leur discussion: «Nous avons eu un échange ferme et franc "; "nous avons clairement fait savoir que les États-Unis voulaient voir des élections en République Démocratique du Congo être organisées en 2018 et que nous n'accepterions plus un nouveau report ${ }^{26}$.

17. Nikki Haley met également à profit son séjour en RDC pour aller réconforter les «survivantes » des violences sexuelles à Kitchanga, au Nord-Kivu. Et, dénonçant les conditions de vie des souffre-douleur des conflits armés à l'Est, elle est plus qu'agressive : «Personne ne devrait vivre comme ça. Nous ne pouvons pas fermer les yeux sur tout cela $»^{27}$.

\footnotetext{
${ }^{21}$ https://www.lepoint.fr/afrique/rd-congo-la-situation-preoccupe-vraiment-les-eveques-catholiques-27-09-2017-2160309_3826.php (19 aout19)

${ }^{22}$ Centre d'Information et d'Animation Missionnaire.

${ }^{23}$ https://www.dw.com/fr/les-usa-peuvent-ils-forcer-joseph-kabila-\%C3\%A0-respecter-le-calendrier-\%C3\%A9lectoral/a-41146363 (15juillet 19)

${ }^{24}$ Ibid.

${ }^{25}$ Union pour la Démocratie et le Progrès Social de feu Etienne Tshisekedi wa Mulumba (père biologique de Fatshi).

${ }^{26} \mathrm{https} / / / \mathrm{cas}-$ info.ca/2017/10/cnn-revele-la-petite-phrase-de-nikki-haley-sur-joseph-kabila-il-est-temps-pour-lui-de-sen-aller/ (15juillet19) ${ }^{27}$ Ibid.
}

Vincent Mukwege Buhendwa, IJSRM Volume 09 Issue 04 April 2021 [www.ijsrm.in] 
18. Revenue à Kinshasa, après une réunion avec le Président de la $\mathrm{CENI}^{28}$, le Pasteur protestant kabiliste, Corneille Nangaa et ses collaborateurs, Nikki Haley avoue avoir été menaçante car "Il était important de montrer que, chaque jour qu'une élection n'est pas organisée dans ce pays, c'est une femme qui se fait violer, c'est des enfants qui sont enrôlés dans les groupes armés et c'est des jeunes filles qui connaissent des grossesses non désirées. Il est important que nous puissions organiser les élections en $2018{ }^{29}$.

19. Sous une menace à peine voilée, Nikki Haley prévient Corneille Nangaa : "Les élections doivent être organisées en 2018 [sinon], la RDC ne doit pas compter sur le soutien des États-Unis et de la communauté internationale $»^{30}$. Au lendemain de son face à face de tous les enjeux avec JKK, Nikki Haley aurait lâché, aux journalistes qui l'accompagnaient dans sa tournée, sa «petite phrase »-assassine- sur JKK : «Il est temps pour lui de s'en aller! $»^{31}$.

20. Invité à une réunion spéciale du Conseil de Sécurité de l'ONU sur la RDC, She Okitundu, Ministre- de son état- des Affaires étrangères et Coopération internationale, s'indigne que des partenaires de la RDC «ressassent, tel un réflexe pavlovien, l'absence de mise en ouvre des mesures de décrispation politique promises », alors que le Gouvernement avait "posé plusieurs actes positifs dans ce domaine ». Aussi martèle-t-il que «le droit de manifester doit être concilié avec le maintien de l'ordre ». ${ }^{32}$

21. Nikki Haley revient sur le respect de la Constitution en RDC : «on ne peut pas permettre un autre délai » pour les élections et se dit préoccupée par la «politisation» de la CENI ; elle insiste à ce que les listes électorales soient nettoyées des fraudeurs et des décès et que la CENI renonce à l'utilisation de la machine à voter (MAV). Quant à la répression des manifestations des opposants par Kinshasa, Nikki Halley juge pareil acte de "complètement inacceptable » et She Okitundu, agacé, a de quoi s'énerver, avant qu'il ne soit recadré par l'Américaine Nikki Halley :

22. «Vous êtes fatigué qu'on parle de la RDC mais il n'y a pas de joie à le faire. Au lieu de protester contre les commentaires négatifs, on apprécierait que le gouvernement congolais agisse et écoute les Congolais au lieu de blâmer les autres » ${ }^{33}$. Revenant sur le dossier sensible de l'assassinat des experts onusiens au Kasaï (RDC), Nikki Haley s'est offusquée de constater que Kinshasa n'avait toujours pas diligenté les enquêtes pour identifier et sanctionner les coupables. Elle en veut à She Okitundu :

23. "Qu'a fait M. Kabila de ma liste ? Rien n'a été fait des actions qu'il fallait entreprendre "; "Please ask M. Kabila what he did with my list! ». («S'il vous plaît, demandez à Monsieur Kabila ce qu'il a fait de ma liste! ${ }^{34}$. La fameuse liste contenait, semble-t-il, des noms et contacts des présumés coupables de l'assassinat de deux experts onusiens au Kasaï et Nikki Haley l'aurait remise à JKK, lors de leur entretien du 27 oct.2017. Fort probable, JKK a « ignoré » ladite liste, autant que l'ONU elle-même !

Nikki Haley aura été la Dame de fer dans cette politique menée par la Communauté internationale contre Kinshasa. Plus encore, elle est apparue comme le prototype du héros tragique chez qui, «le pouvoir se confond avec le vouloir : il suffit d'ordonner ». Chez des héros de ce calibre, la parole est un acte : dire, c'est faire »; et avec Nikki Haley, on a finalement compris qu' «on réussit chez les rois les expériences qui ne réussissent jamais chez les humbles ${ }^{35}$.

\section{III.3. Laurent Cardinal Monsengwo croise le fer avec les médiocres}

24. Laurent Cardinal Monsengwo, lors de sa messe de minuit du 24 déc. 2015 : "Il est révolu le temps où l'on cherchait à conserver le pouvoir par les armes, en tuant son peuple. Celui qui respecte la Constitution n'a rien à craindre de la justice ». Pendant ce temps, en sept. et déc. 2015, les manifestations des partisans du non-glissement sont réprimées dans le sang ${ }^{36}$.

\footnotetext{
${ }^{28}$ Commission Electorale Nationale Indépendante.

${ }^{29}$ Ibid.

${ }^{30}$ Ibid.

${ }^{31}$ Ibid.

${ }^{32}$ https://cas-info.ca/2017/10/cnn-revele-la-petite-phrase-de-nikki-haley-sur-joseph-kabila-il-est-temps-pour-lui-de-sen-aller/ (15juillet19)

33 Ibid.

${ }^{34}$ Ibid.

35 J.-D.MALLET, La tragédie et la comédie p.67.

${ }^{36}$ https://www.voaafrique.com/a/que-les-mediocres-degagent-mgr-monsegwo-la-bete-noire-de-kabila/4196787.html (4aout19)
} 
25. En janv. 2018, le prélat précité lâche sa bombe verbale : «il est temps que les médiocres dégagent et que règnent la paix et la justice en $R D C »^{37}$.

26. Le Cardinal use de bien d'autres mots provocateurs pour qualifier/disqualifier le régime, notamment le «mensonge systémique ».

27. Aussi s'en prend-il aux agissements des «prétendus vaillants hommes en uniforme » qui traduisent malheureusement «la barbarie ${ }^{38}$. Et d'en ajouter, le 4 janv. 2018 :

28. «Il est temps que la vérité l'emporte sur le mensonge systémique, que les médiocres dégagent et que règnent la paix, la justice en République Démocratique du Congo» ${ }^{39}$. Le mot d'ordre « dégage ! » semble donné et Sonia Rolley de la $\mathrm{RFI}^{40}$ l'assimile à celui lancé par des manifestants tunisiens qui avaient réussi à mettre en déroute Ben Ali en $2011^{41}$.

29: Dans un communiqué du 2 janv.2018, Mgr Luis Mariano Montemayor, Nonce Apostolique du Saint Siège en RDC, est d'avis que : "La promotion de la justice sociale et la défense des droits civils et politiques des citoyens font intégralement partie de la doctrine sociale de l'Eglise ${ }^{42}$.

30. Ainsi appuyé, le CLC appelle les Congolais à «libérer l'avenir de la RDC et réclamer l'application de l'Accord de la Saint-Sylvestre dans son intégralité ${ }^{43}$.

31. A l'issue du Conseil des ministres du 5 janv. 2018, le Gouvernement de la RDC évoque la menace des poursuites judiciaires contre l'Archevêque de Kinshasa : "Monseigneur Laurent Monsengwo a tenu des propos injurieux à l'endroit des dirigeants du pays ainsi que des forces de l'ordre» ${ }^{44}$.

32. Le 7 janv.2018, Justin Bitakwira, Ministre du Développement rural, plus opportuniste que kabiliste, prend le relais : "On doit stopper le Cardinal Monsengwo comme on a stoppé Muanda Nsemi ${ }^{45}$... Parce que quand vous l'entendez parler, c'est la voix d'un putschiste qui a raté son coup. On ne doit pas tout permettre dans ce pays... ${ }^{46}{ }^{4}$,menace-t-il de tout son poids !

En traitant Laurent Cardinal Monsengwo de «putchiste », comparé à Muanda Nsemi, J. Bitakwira fait usage de l'épiplexis, une figure de style consistant à blâmer un accusé pour faire apparaitre ses fautes, en même temps qu'on étale les peines auxquelles il s'expose ${ }^{47}$. Cependant, les propos de J. Bitakwira rejoignent l'opinion de nombreux Congolais qui estiment que les Hommes de Dieu sont très enthousiastes à malmener les autorités politiques et administratives corrompues de leur pays. Et pourtant, à y regarder de près, pour la plupart d'entre eux, la bonne gouvernance dont ils sont apôtres est paradoxalement invisible dans les institutions ecclésiastiques qu'ils dirigent...

\section{III.4. Les Protestants entrent dans la danse et protestent...}

33. Le 16 janv.2018, Mgr François-David Ekofo, Evêque protestant, s'adresse aux dignitaires du régime, en la Cathédrale du Centenaire (Kinshasa) lors d'un culte religieux en mémoire de Mzee Laurent-Désiré Kabila $^{48}$. Il transforme sa prédication en un véritable rappel à l'ordre ${ }^{49}:$ "J'aime bien l'athlétisme où il y a des courses à pied surtout. Et j'aime spécialement une course : la course de relais où une personne transmet le bâton à une $2^{e}$ personne, à une $3^{e}$ et à une $4^{e}$. Dans l'histoire du pays, c'est pareil aussi »; (En RDC), j'ai l'impression que l'État n'existe pas vraiment. L'État n'existe pas réellement », tout en stigmatisant la mauvaise gestion des richesses de la RDC, "un pays à qui Dieu a tout donné mais aux populations pauvres ». Car, selon lui, «Dieu a donné la gestion du Congo à nous Congolais. Et c'est nous Congolais

\footnotetext{
${ }^{37}$ https://www.radiookapi.net/2018/01/02/actualite/politique/cardinal-monsengwo-il-est-temps-que-les-mediocres-degagent-et-que (4 aout19)

${ }^{38}$ https://www.francetvinfo.fr/monde/afrique/politique-africaine/rdc-laurent-monsengwo-l-archeveque-qui-veut-degager-le-mediocre-kabila_3055523.html (4aout19).

${ }^{39}$ Ibid.

${ }^{40}$ Radio France internationale.

${ }^{41}$ Ibid.

${ }^{42}$ https://www.radiookapi.net/2018/01/02/actualite/politique/marche-des-laics-en-rdc-la-nonciature-dit-en-avoir-informe-le-vatican (4 aout19)

${ }^{43}$ Ibid.

${ }^{44}$ https://www.francetvinfo.fr/monde/afrique/politique-africaine/rdc-laurent-monsengwo-1-archeveque-qui-veut-degager-le-mediocre-kabila_3055523.html (4aout19)

${ }^{45}$ Un gourou congolais soi-disant opposant au régime, arrêté, embastillé puis évadé, au nez et à la barbe des autorités de Kinshasa.

${ }^{46}$ Ibid.

${ }^{47}$ MUTUNDA Mwembo, Eléments de logique, Médiaspaul, Kinshasa, 2006, p.102.

$483^{\mathrm{e}}$ Président de la RDC, Lumumbiste conduit au pouvoir par l'Alliance des Forces Démocratiques pour la Libération (du Congo)/ AFDL et mort assassiné à Kinshasa, le 16 janvier 2001.

${ }^{49}$ https://cas-info.ca/2018/01/leglise-protestante-du-congo-demande-poliment-a-joseph-kabila-de-transmettre-le-baton-a-un-autre/ (4 aout19)
} 
qui, un jour, rendrons compte à Dieu de notre gestion de sa terre, de la RDC ${ }^{50}$ Et de conclure : «Même Dieu lui-même se demande comment nous sommes pauvres $» 51$.

34. Ce message fait suite à celui de la CENCO qui ne cesse de crier au loup : "Le pays va très mal. Debout, Congolais!».

35 : L'Evêque protestant exhorte le peuple congolais «à prendre en main son destin commun » et à "avoir une présence active et courageuse dans le monde de la politique $\gg^{52}$. La prédication de l'évêque protestant fait mouche!

. Cette stratégie vise également à mettre l'adversaire dans une position gênante, celle de la relation inégalitaire qui s'établit ainsi, assurant au vainqueur une position avantageuse, en éloignant le plus souvent le public-témoin de son adversaire ${ }^{53}$. L'engagement de Laurent Cardinal Monsengwo, de François-David Ekofo et de bien d'autres leaders chrétiens de Kinshasa, a semblé risqué, susceptible de mettre leur vie en danger, là où d'autres leaders religieux congolais, peu courageux, même au sein de la CENCO, se résignaient et exhortaient leurs fidèles à organiser des neuvaines et veillées de prière pour la paix en RDC. Lâcheté oblige !

\section{III.5. Rêve d'une transition sans Kabila, Yebela contre Wumela, Prix Nobel de la paix...}

36. Les partisans du non-glissement piaffent d'impatience et commencent à mijoter la probabilité d' "une transition sans Kabila» pour une durée de six mois ou une année. Cela permettrait aux autorités intérimaires de conduire réellement le peuple congolais vers des élections apaisées, transparentes, crédibles et démocratiques.

37. La paternité de l'idée «une transition sans Kabila », géniale mais infructueuse, revient à la LUCHA, à travers sa figure de proue, Jean-Pierre Mulumba.

38: LUCHA lance une autre campagne dénommée «Bye Bye Kabila!» qui ne produit pas moins d'effets, bien que, au cours des manifestations, nombreux de ses membres sont raflés par les agents de l'ordre et écroués ; «Kabila dégage !»; «Tolembi ye ! (Nous avons marre de lui !) ; «Game is over !» (la partie est terminée !) ; «Transition sans Kabila »; «Yebela !»(Tu est prévenu !).

39. Les pro-glissement affichent aussi «Wumela » (règne à jamais !) contre Yebela !: deux Professeurs d'université «Kabilistes » défraient la chronique avec leurs faits et gestes qui frisent le ridicule : (i) Henri Mova Sakanyi et (ii) Tryphon Kin-Key Mulumba sont évoqués. ${ }^{54}$. Le dernier organise des campagnes de soutien à l'Autorité morale (JKK) et déclare, sans froid aux yeux : "J'ai créé Kabila Désir pour réhabiliter un homme et ses œuvres », tandis que le premier tient des séances de soutien à JKK au cours desquelles il rampe, bon enfant, tel un reptile ou un batracien, scandant : «Mpo na Raïs, tokonguluma ! , c'est-à-dire «Pour le Président, nous hurlerons ! ». Deux Renards alléchés par l'odeur du fromage de Sir le Corbeau dans les fables de Jean de Lafontaine. Le moins qu'on puisse dire.

40. Laurent Cardinal Monsengwo et Dr Denis Mukwege sont pressentis candidats à la succession de JKK. À ce sujet, un cacique du régime confie à Jeune Afrique : "C'est surtout une rêverie de plus de Didier Reynders (Ministre belge des Affaires étrangères)... Après avoir rêvé que l'Angola ou le Rwanda enverraient des troupes pour nous renverser, il pousse Mukwege vers une voie sans issue. "Et de conclure : " S'il doit un jour y avoir une transition sans Kabila, nous n'accepterons jamais qu'elle soit conduite par le docteur Mukwege, encore moins par le Cardinal Laurent Monsengwo ${ }^{55}$.

41. L'homme qui répare les femmes de Colette Braeckman ${ }^{56}$ est aussi hostile au régime de JKK que Laurent Cardinal Monsengwo,et les deux n'ont pas leurs langues dans la poche.

42. Dr Denis Mukwege a été Proclamé Prix Nobel de la Paix avec Nadia Murad, le 5 oct. 2018 et cette nouvelle «a pourtant été accueillie froidement à Kinshasa ${ }^{57}$ parce qu'au cours de ces dernières années,

\footnotetext{
${ }^{50}$ Ibid.

${ }^{51}$ Ibid.

${ }_{53}^{52}$ https://www.lepoint.fr/afrique/rdc-comite-laic-de-coordination-ce-fer-de-lance-de-la-contestation-31-05-2018-2223010_3826.php (15juillet19)

${ }_{53}$ M.SANI, Op.Cit.p.78.

${ }^{54}$ https://www.radiookapi.net/2016/02/23/emissions/le-grand-temoin/tryphon-kin-kiey-mulumba-jai-cree-kabila-desir-pour-rehabiliter (15 juillet19).

${ }^{55}$ Jeuneafrique $\mathrm{n}^{\circ} 3014$ du 14 au 20 oct.2018, p.26

${ }^{56}$ C. BRAECKMAN, L’homme qui répare les femmes, Ed.André Versaille, 2012

${ }^{57}$ Jeuneafrique $\mathrm{n}^{\circ} 3042-3043$ du 28 avril au 11 mai 2019. p. 12 .
} 
Dr Denis Mukwege n'a cessé d'exiger la démission de JKK et de son « groupe de copains corrompus » ${ }^{58}$. Depuis qu'il est sorti du bois, à la suite des atrocités des violences sexuelles massives, liées aux conflits armés et prises en charge par son hôpital de Panzi à Mushununu, la renommée de ce fils d'un pasteur protestant pentecôtiste est allée au-delà de son Kivu natal, de la RDC et de l'Afrique. La simple évocation de son nom n'a cessé de causer de l'insomnie aux caciques du régime de Kinshasa.

43. En 2014, Dr Denis Mukwege a reçu de l'Union Européenne (UE) le Prix Sakharov. La même année, JKK nomme Jeanine Mabunda Mudiayi Lioko, l'une des femmes de fer du régime et «anti-Mukwege » ${ }^{59}$, sa Conseillère spéciale en matière de lutte contre les violences sexuelles. La présidente de l'Assemblée nationale avait alors pour mission de lutter contre ce qu'elle a appelé, en 2015, «l'ONGisation » des violences sexuelles en RDC que Kinshasa reproche au Prix Nobel congolais ${ }^{60}$.

44. Le Professeur Abbé Richard Mugaruka est décédé le 7 nov.2016. Il a laissé derrière lui un combat inachevé, mais fortement engagé contre le régime de JKK et pour l'alternance politique en RDC. AntiKabila et pro-Mukwege, il n'a cessé, de son vivant, de malmener le régime et haranguer les jeunes pour qu'ils se prennent en charge. Ses propos étaient constants : "un peuple qui n'a pas de raisons de mourir, n'a pas non plus de raisons de vivre!». Son décès prématuré fut une perte pour les anti-glissement et antiKabila.

\section{III.6. Joseph Kabila sort de son mutisme: «le Congo n'est pas une république bananière !»}

45. Dans sa conférence de presse du 26 janv. 2018, JKK sort de son mutisme et déclare : «Je constate depuis plusieurs années qu'on vous a tout dit. Alors tout. Sauf la vérité... » ${ }^{61}$. Ses flèches verbales sont orientées vers la CENCO : «Jésus-Christ n'a jamais présidé une Commission électorale(...). Rendons à César ce qui est à César, et à Dieu ce qui est à Dieu. Quand on essaie de mélanger les deux, c'est dangereux. Le résultat est toujours négatif ${ }{ }^{62}$

46. JKK réitère son engagement de conduire le pays vers des élections en 2018, en dépit d' "une résistance farouche de la part d'une frange de l'opposition, d'une frange de ce qu'on appelle la société civile, de la part d'un homme de Dieu ». Là, il s'agit, bel et bien, de Laurent Cardinal Monsengwo, pour ne pas le citer! Et d'ajouter : "Malgré le bon déroulement des élections de 2006, il y avait des contestations : le perdant et une partie de l'Eglise ${ }^{63}$.

47. En dépit de cette résistance, il entrevoit l'organisation «des élections libres et démocratiques, des élections organisées par les Congolais. Et pas des élections influencées par quelqu'un, un pays, ou un couple de pays» sans jamais préciser s'il est, oui ou non, candidat à ces dernières.

48. A la question «serez-vous candidat à la présidentielle du 23 décembre prochain ? » posée par une journaliste, il demande à ce qu'un exemplaire de la Constitution soit remis à cette dernière. Or la même Constitution prévoit la tenue des élections en 2016 et, deux ans plus tard, celles-ci n'ont toujours pas eu lieu !

49. Au sujet des mesures de décrispation prônées par l'Accord de la Saint-Sylvestre, JKK s'interroge : "Quand on parle de la décrispation, de quoi est-ce qu'il s'agit? Est-ce qu'il faut libérer 10000 prisonniers, pour constater qu'effectivement il y a une décrispation? Est-ce qu'il faut qu'on ait 3000 partis politiques et là on sera content, c'est la décrispation ? Le Congo n'est pas une république bananière ». ${ }^{64}$

\footnotetext{
${ }^{58}$ Ibid.

${ }^{59}$ Jeuneafrique n$^{\circ} 3042-3043$ du 28 avril au 11 mai 2019.p.12.

${ }^{60} \mathrm{Ibid}$.

${ }^{61}$ http://www.rfi.fr/afrique/20180126-rdc-kabila-prend-parole-presse-5-ans-silence (15 juillet19)

62 Ibid.

${ }^{63}$ Ibid.

64 Ibid.
} 
50. Sur un ton voilant frustration et menace, il s'en prend aux meneurs de la Communauté internationale : «Ceux qui ont assassiné la démocratie dans ce pays devraient faire preuve d'humilité en lieu et place de donner des leçons $\gg .{ }^{65}$

51.JKK clame la souveraineté nationale: «Mon pays n'est pas à vendre, la RDC doit être respectée ». ${ }^{66}$

52. Dans cette menace, la MONUSCO ${ }^{67}$ n'est pas oubliée : «Parce qu'après une vingtaine d'années, on a comme l'impression que c'est une mission qui a comme ambition de rester. Mais rester jusqu'à quand? Si l'ambition c'est de considérer le Congo sous tutelle des Nations unies... On a toujours posé la question à nos amis de la MONUSCO: "citez-nous un seul groupe (armé) que vous avez réussi à maîtriser, à éradiquer, puisque c'est ça le mot ?" Aucun. » ${ }^{68}$.

\section{III.7. Locomotive électorale et douleurs d'enfantement d'une alternance politique inespérée...}

53. Au 29 janv.2018, la CENI a enregistré 46 millions d'électeurs dont 24254196 hommes et 21790257 femmes.

54. A l'issue de son Assemblée Générale du 15 au 17 fév. 2018, la CENCO s'en prend au régime qui mène une «série de campagnes d'intoxication, de dénigrement, voire de diffamation, visant à affaiblir la force morale de l'Eglise, particulièrement de Son Eminence Laurent Cardinal Monsengwo, et à détourner l'attention du Peuple de vrais enjeux ${ }^{69}:$ «Nous réaffirmons notre soutien et notre proximité au Cardinal Archevêque de Kinshasa. Inébranlables dans notre foi en Jésus-Christ, Roi de l'univers, et fidèles à notre mission prophétique, nous n'abandonnerons jamais notre engagement pour l'avènement d'un État de droit en République démocratique du Congo $\gg^{70}$.

55. Le 23 fév.2018, Laurent Cardinal Monsengwo célèbre une messe en mémoire des victimes fauchées par les agents de l'ordre lors de récentes manifestations à Kinshasa et dans les provinces. Monsieur l'Abbé François Luyeye est formel : "La marche des chrétiens ne s'arrêtera pas »; "Les initiatives du Comité Lä̈c de Coordination sont à applaudir, et nous en attendons d'autres ${ }^{71}$.

56. Le 14 fév.2018, une réunion tripartite est tenue à Kinshasa entre les Présidents Joseph Kabila (RDC), João Laurenço (Angola) et Denis Sassou Nguesso (Congo-Brazza), les deux derniers représentant respectivement la $\mathrm{SADC}^{72}$ et la $\mathrm{CIRGL}^{73}$.

57. Le 17 fév.2018, JKK se rend à Lusaka pour un entretien avec son homologue zambien Edgar Lungu. Personne ne sait de quoi ils ont parlé.

58. Revenu à Kinshasa, JKK reçoit en audience l'émissaire angolais, Manuel Domingos Augusto, Ministre des Affaires étrangères. Est-ce pour contraindre JKK à renoncer au $3^{\mathrm{e}}$ mandat ?

59. Le 22 fév.2018 le Président gabonais, Ali Bongo, arrive à Kinshasa et s'entretient avec le Président Joseph Kabila.Même mission, même objectif.

60. Le 26 fév.2018, déclaration musclée du Ministre botswanais des Affaires étrangères, dénonçant le fait que le Président Joseph Kabila cherche à se maintenir au pouvoir. Etonnant!

61. Le Président botswanais, Ian Khama, quitte le pouvoir fin mars 2018.

62. La pression régionale et internationale s'accroît sur Kinshasa pour « le pousser dans la bonne direction », c'est-à-dire le contraindre au respect du calendrier électoral qui exclut aussi le $3^{\mathrm{e}}$ mandat $^{74}$. Le taux se resserre sur les Kabilistes qui, jusque-là, n'y croient pas de leurs yeux.

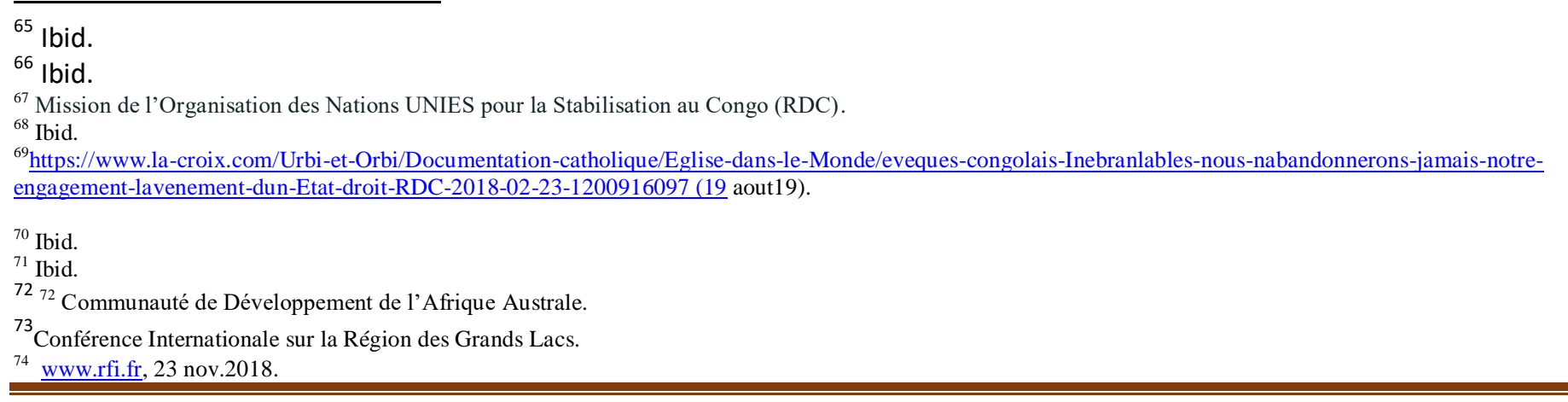


63. Le 27 fév.2018, le Président zimbabwéen, Emmerson Mnangagwa, s'entretient avec Joseph Kabila. De plus en plus, l'opinion croit que JKK est plus que jamais pressé de toutes parts pour respecter la Constitution, et le calendrier électoral, et-surtout-renoncer au $3^{\mathrm{e}}$ mandat $^{75}$.

64. Du 9 au 12 mars 2018, les partisans de Moïse Katumbi se réunissent en République Sud-Africaine et forment une nouvelle plate-forme de l'opposition dénommée « Ensemble pour le changement », Cette plateforme donnera plus tard naissance à la coalition «Lamuka» en Swahili et Lingala et qui signifie, en français, «Réveillez-vous »!.

\section{III.8. Feuilleton Mö̈se Katumbi, Machine à voter, Insécurité chronique à l'Est et renoncement au $3^{e}$ mandat}

65. Le feuilleton Moïse Katumbi a défrayé la chronique de mars 2015 à juin $2018^{76}$ : (1) le 23 juin 2015 , une plainte est déposée contre lui pour « fraude douanière » et pour laquelle rien ne lui a été notifié ; (2) le 24 avril 2016, il est inculpé d'atteinte à la sûreté de l'Etat, dans une affaire de recrutement des mercenaires, dont l'Américain Darryl Lewis ; (3) le 20 juin 2016, il est condamné en première instance dans l'affaire de spoliation d'un immeuble de Sieur Alexandros Stoupis, à Lubumbashi ; (4) le 22 mars 2018, il est soupçonné d'usurpation de la nationalité congolaise ; (5) le 20 avril 2018, il est soupçonné de financer un déserteur de l'armée, l'ex-officier des FARDC, Col. John Tshibangu qui menaçait le régime de Kinshasa ; (6) le 23 avril 2018, il est soupçonné de complicité avec une certaine Brigitte Safari Misabiro (de nationalité rwandaise), détentrice d'un document subversif relatif à la création d'un mouvement insurrectionnel en RDC ; (7) le 14 juin 2018, il est soupçonné d'utiliser un passeport (congolais) invalide, alors qu'il est en exil en Belgique. Ce harcèlement judiciaire a été, en lui-même, suffisant pour barrer la route à la présidentielle à cet opposant d'obédience catholique, très proche de la CENCO.

66. La «Machine à voter », de fabrication sud-coréenne, demeure un «casus belli » entre le régime et la Société civile, les opposants et leurs alliés. Elle est débaptisée par ces derniers «Machine à voler»; « Machine à fauter »; « Machine à tricher ».

67. Le $1^{\text {er }}$ avril 2018, le célèbre Antoine Agbepa Mumba, dit Koffi Olomidé, récupère, à son tour, le débat $^{77}$ et s'insurge contre cette machine controversée : "J'ai sillonné l'Europe, le monde mais je n'ai jamais entendu parler de cet outil des élections. Pourquoi voulez-vous être pionniers en expérimentant une chose qui peut rendre la tâche difficile au pays ? " ${ }^{78}$. L'icône de la chanson congolaise venait de jeter un pavé dans la marre. Il en fut certes très applaudi, mais nullement écouté par les Kabilistes !

68. Le 22 mai 2018, Jean Cyrus Mirindi Batumike Nkuba, soi-disant chercheur congolais en droit-et en droit constitutionnel congolais-, déclare sur les médias locaux : «Il reste un mandat à Joseph Kabila pour atteindre le plafond constitutionnel ». Motu proprio, il décide que «Le comptage qu'on doit prendre-en compte- constitutionnellement, c'est le comptage qui débute à partir de l'élection de 2011. A partir de ce moment-là, le Président de la République a fait un mandat et il lui reste un deuxième mandat pour atteindre le plafond constitutionnel. Ça fait mal mais c'est une réalité ${ }^{79}$. Ça fait vraiment mal ! Jean Cyrus Mirindi est un héritier idéologique d'Evariste Boshab, ancien Conseiller politique de JKK et Ministre de l'Intérieur, ancien «Speaker » de la Chambre basse du Parlement, ardent promoteur de la révision constitutionnelle afin de voir son «Autorité morale » accéder au $3^{\mathrm{e}}$ mandat. Au sujet de la sortie médiatique pro-glissement de Jean Cyrus Mirindi, les anti-glissement dénoncent une « interprétation intéressée »-et combien erronée- de la Constitution.

69. Charles Mwando Nsimba de $1^{\prime} \mathrm{UNADEF}^{80}$ : «les gens veulent tout simplement créer la panique et désorganiser l'Etat. Mais nous, nous demandons simplement à Kabila ${ }^{81}$ de respecter notre Constitution, s'il

\footnotetext{
75 www.rfi.fr, 23 nov.2018.
}

${ }^{76}$ https://www.jeuneafrique.com/549734/societe/rdc-katumbi-et-1-affaire-des-mercenaires-justice-et-services-de-renseignements-se-contredisent/ (1 ${ }^{\text {er }}$ aout 19$)$.

${ }^{77}$ En Swahili on dit « kulangula » dossier, c'est-à-dire « récupérer, acheter en gros », etc.

${ }^{78}$ https://fr.africanews.com/2018/10/04/en-rdc-koffi-olomide-s-insurge-contre-la-machine-a-voter/ (26 aout19).

${ }^{79}$ https://www.mediacongo.net/article-actualite-38532 cyrus mirindi il_reste un mandat_a joseph kabila pour atteindre le plafond constitutionnel.html (11sept19).

${ }^{80}$ Union Nationale des Démocrates Fédéralistes.

${ }^{81}$ Dans leurs attaques, les détracteurs de JKK ne le ménagent plus et ne cessent de l'apostropher, parce qu'à leurs yeux le Président sortant a perdu toute légitimé et légalité. 
lui reste encore un peu de conscience $»^{82}$. Ici, le Président Joseph Kabila est désormais considéré, par ses détracteurs et le gros du peuple congolais, comme un citoyen lambda. Et désormais, il devra se plier à ce verdict populaire et se comporter ainsi.

70. Georges Kapiamba de l'ACAJ ${ }^{83}$ prévient le régime : «Il est temps que nos frères et sœurs de la Majorité présidentielle abandonnent cette stratégie dangereuse. Parce que, là, ils visent tout simplement à déstabiliser encore ce pays qui peine à se remettre ${ }^{84}$.

71. Prof. Jacques Djoli, constitutionnaliste de son état et cadre du MLC ${ }^{85}$, enfonce le clou : «La Constitution est un défi vis-à-vis de l'arbitraire, vis-à-vis de l'intemporalité, vis-à-vis de la mentalité du pouvoir. La Constitution vient limiter le pouvoir dans le temps et dans l'espace. D'ailleurs nous devons continuer à travailler pour que ce que nous appelons des démagogues et les délinquants constitutionnels ${ }^{86}$ ne puissent pas amener ce pays dans les affres de la guerre civile ${ }^{87}$.

72. Le 8 juin 2018, pour lui faciliter la course à la présidentielle de la RDC, la CPI $^{88}$ diligente la libération de Jean-Pierre Bemba. Sa candidature sera plus tard invalidée par la CENI, invalidation confirmée par la Cour Constitutionnelle. Jean-Pierre Bemba s'en plaindra en ces termes : "Kabila a choisi ses propres opposants »; «Kabila a eu peur de moi $»^{89}$.

73. Le 22 juin 2018, Kinshasa refuse de renouveler l'accréditation de Sonia Rolley, Envoyée spéciale permanente de la RFI à Kinshasa.

74. RFI est une station-radio massivement écoutée en RDC, contrebalançant les nouvelles diffusées par la $\mathrm{RTNC}^{90}$.

75. Les anti-glissement sont d'avis que la RTNC sert de caisse de résonnance au régime de Kabila. Selon eux, «les seules vraies informations diffusées par la RTNC sont les 'communiqués nécrologiques' »!

76. Le 4 juillet 2018, Laurent Cardinal Monsengwo s'en prend aux « Satanistes ». Là, il cible bel et bien les gouvernants et mandataires publics qui pillent la RDC : "Quand tu voles l'argent public et tu deviens riche alors que le peuple souffre, tu es sataniste ».

77. Il menace et maudit les dignitaires du régime : "Tôt ou tard, tu vas payer cela. Car Dieu n'est pas injuste. Ce sont ceux qui ont un cour droit que Dieu bénit, car ceux qui volent l'argent de l'Etat et ils sont heureux avec leurs biens mal acquis, Dieu finira par les punir ». Qui dit mieux?

78. Il exhorte les jeunes et les mobilise pour le combat: "Quand rien ne marche dans ton pays, quand un individu veut prendre le pouvoir par la force, et veut prendre plus de 80 millions d'habitants en otage, tu as le droit de te lever et combattre cette dictature. C'est ton devoir patriotique, toi chrétien! Un chrétien est une personne de justice $»^{91}$.

79. Le 19 juillet 2018, JKK s'adresse aux membres des deux Chambres du Parlement réunies en Congrès. Il ne dit rien sur son avenir politique, les anti-glissement montent au créneau :

(1) Augustin Kabuya, Porte-Parole de l'UDPS ${ }^{92}$ : «Le peuple congolais n'a plus rien à attendre d'une personne qui se croit monarque éternel ${ }^{93} ;(2)$ un autre opposant : «Nous sommes déçus par ce discours du président Kabila qui a encore raté l'occasion de se prononcer sur son avenir politique ${ }^{94}$; (3) Ida

\footnotetext{
${ }^{82}$ https://www.mediacongo.net/article-actualite-38532_cyrus_mirindi_il_reste_un_mandat_a_joseph_kabila_pour_atteindre_le_plafond_constitutionnel.html (11sept19).

${ }^{83}$ Association Congolaise pour l'Accès à la Justice.

${ }^{84}$ https://www.mediacongo.net/article-actualite-38532_cyrus_mirindi_il_reste_un_mandat_a_joseph_kabila_pour_atteindre_le_plafond_constitutionnel.html (11sept19).

${ }_{86}^{85}$ Mouvement de Libération du Congo.

${ }^{86}$ Ici, il cite, sans les nommer, Jean Cyrus Mirindi Batumike Nkuba, Evariste Boshab, et tous les Kabilistes confondus.

${ }^{87}$ https://www.mediacongo.net/article-actualite-38532_cyrus_mirindi_il_reste_un_mandat_a_joseph_kabila_pour_atteindre_le_plafond_constitutionnel.html (11sept19).

${ }^{88}$ Cour Pénale Internationale.

${ }^{89}$ Jeune Afrique $\mathrm{n}^{\circ} 3034$ du 3 au 9 mars 2019.p.23

${ }^{90}$ Radio Télévision Nationale Congolaise.

${ }^{91} \mathrm{https} / / / \mathrm{www}$. lephareonline.net/apres-mediocres-cardinal-monsengwo-denonce-satanistes/ (4aout19).

92 Union pour la Démocratie et le Progrès Social.

${ }^{93} \mathrm{https}: / /$ www.voaafrique.com/a/kabila-va-annoncer-prochainement-des-d\%C3\%A9cisions-importantes-selon-guterres/4480196.html (22 août19).

${ }^{94}$ Ibid.
} 
Sawyer, Directrice Afrique de $\mathrm{HRW}^{95}$ et «persona non grata en RDC pour ses prises de position antérieures : "Kabila n'a rien dit sur son avenir politique. Toujours pas de signe clair qu'il prévoit de quitter le pouvoir conformément à la Constitution (et) permettre un scrutin crédible. Il faut d'urgence une pression accrue pour le convaincre de changer de cap ${ }^{96}$.

80. LUCHA : "Candidat? Levons-nous tous aussitôt pour le démettre comme on aurait dû le faire depuis longtemps. Pas candidat? Continuons à exiger de vraies élections, impossibles avec lui et sa CENI ${ }^{97}$ aux commandes" $^{\prime \prime 8}$.

81. A l'Est du pays, l'insécurité chronique perpétrée par les soi-disant ADF-Nalu ${ }^{99}$ ne baisse pas d'un cran et l'épidémie de la Maladie à Virus Ebola (MVE, une autre MAV ?) s'est aussi déclarée à Beni, Butembo, Ituri La rumeur du complot international se répand que le continent noir, dans son entièreté, est menacé par la guerre bactériologique d'origine occidentale.

82. Mgr Melchisédech Sikuli Paluku, Evêque de Butembo-Beni, interpelle le régime : «Alors que nous sommes engagés sur le front de la riposte contre Ebola, voici que nous venons d'affronter, une fois de plus, des attaques sanglantes dont notre population de BENI a été victime samedi 22 septembre 2018.Ces attaques ont entraîné la mort de 18 personnes, dont 14 civils et 4 militaires, et 9 blessés dont 6 civils et 3 militaires, d'après les média, et la désolation des populations nous afflige et nous préoccupe profondément»; «Ne devrait-on pas changer de stratégie et des méthodes d'investigations pour mettre définitivement fin à cette barbarie qui semble ne pas dire son nom? ${ }^{100}$ ». Face à cette détresse inouïe, l'opinion recherche un bouc-émissaire. Car Dieu n'est pas si cynique pour déverser sur la RDC et, en même temps, les ADF et Ebola. Du coup, la théorie du complot refait surface, corroborée par l'invention macabre du VIH/Sida (1960-70) par Dr Hilary Koprowski pour dépeupler la RDC et ses voisins mais qui a occasionné des milliers de victimes collatérales. Et Ebola est de retour en RDC et en Afrique.

83. Le 23 oct.2018, l'UDPS de Fatshi fait défection avec ses pairs de l'opposition et annonce qu'elle ira aux élections, avec ou sans la MAV. Jean-Marc Kabund, son Secrétaire Général, est formel: "Dire qu'il ne faut pas aller aux élections avec la machine à voter, (...) ce serait tomber dans le piège de Kabila (...). Entre le report des élections pour raison de machines à voter et aller aux élections avec ou sans machines, l'UDPS a choisi le moindre mal, c'est d'aller aux élections et de consacrer par-dessus tout le départ de Joseph Kabila du pouvoir ${ }^{101}$. Une prophétie ?

84. La Mouvance Présidentielle a deux fers au feu et doit s'organiser et se choisir un dauphin.

85. Le 9 mai 2018: Joseph Kabila lâche enfin, en désignant Emmanuel Ramazani Shadary : «Notre candidat, il est là !» ${ }^{102}$.

86. Le néologisme «dauphinisation» entre dans le lexique politique congolais. Emmanuel Ramazani Shadary est enfin "dauphinisé ». Les anti-glissement se frottent les mains ! Ils voient en ce dauphin un cheval boiteux qui n'ira pas loin.

87. Toutefois, pro et anti-glissement se rapprochent davantage : en désignant son dauphin, JKK annonce publiquement qu'il n'est plus candidat à sa propre succession. Personne n'y croyait jusque-là ! Sauf peutêtre Lambert Mende et André-Alain Atundu (A3).

88. Le13 août 2018, A3 avait soutenu, sous un ton moqueur : "le Chef de l'Etat avait pris l'engagement devant la nation que la Constitution serait respectée, un engagement renouvelé dans l'accord du 31 décembre 2016, qui dispose que le président ne pourra solliciter un troisième mandat. J'estime que, pour

\footnotetext{
${ }^{95}$ Human Rights Watch.

${ }^{96} \mathrm{https} / / /$ www.voaafrique.com/a/kabila-va-annoncer-prochainement-des-d\%C3\%A9cisions-importantes-selon-guterres/4480196.html

(22 août19).

${ }_{98}^{97}$ Commission Electorale Nationale Indépendante.

${ }^{98}$ Ibid.

99 Allied Democratic Forces": groupe terroriste (armé)d'origine ougandaise, instrumentalisé et opérant sur le sol congolais tout en prétendant restaurer la démocratie en Ouganda.

100 https://4pouvoir.cd/2018/09/massacres-a-beni-mgr-sikuli-melchisedech-appelle-le-gouvernement-congolais-a-changer-des-methodes-d-investigations/ aout19)

${ }^{101}$ http://www.rfi.fr/afrique/20181023-rdc-udps-machine-voter-jean-marc-kabund (15juillet19).

${ }^{102}$ https://www.jeuneafrique.com/mag/614521/politique/presidentielle-en-rd-congo-ramazani-shadary-le-medvedev-de-kabila\%e2\%80\%89/ (2sept19).
} 
des esprits normalement constitués, cela aurait dû suffire ${ }^{103}$. A3 se fait ainsi le luxe de railler ses adversaires. Aliis verbis, les opposants sont des «esprits anormalement constitués ", c'est-à-dire des étourdis. Des stupides, pourtant sceptiques.

89. Renoncement de JKK au $3^{\mathrm{e}}$ mandat : un acte positivement salué par l'opinion nationale et internationale. En sacrifiant ainsi son égo, JKK donne l'air d'avoir revêtu le manteau du héros cornélien qui trouve la force et les moyens de surmonter les coups du sort, à l'instar de l'Empereur Auguste qui «ne sait quelle attitude adopter face aux tentatives d'assassinat dont il fait l'objet. Doit-il toujours réprimer ? Mais les comploteurs deviennent aussitôt des martyrs de la cause républicaine et de nouveaux conspirateurs se lèvent. Il ne peut pas pour autant se laisser assassiner. Auguste finira par comprendre que, pour que Rome change, il lui faut d'abord se changer lui-même, devenir un modèle de magnanimité. Il pardonnera à tous »104.

90. La CENI et la Cour Constitutionnelle servent alors de filtres et de tremplins institutionnels, y compris l'ANR ${ }^{105}$, pour accéder au pouvoir, avec ou sans la MAV. Au total, 25 candidats, dont une femme, à la présidentielle ont été enregistrés par la CENI, moyennant une caution non remboursable de $100000 \$$ USjamais en francs congolais, dollarisation oblige !-, Pour la plupart des candidats politiciens, pareilles dépenses sont couvertes par l'argent volé au trésor public- donc au peuple congolais- et recyclé dans le processus électoral.

91. Le 25 août 2018, la CENI « invalide » (rejette) six (6) candidatures à la présidentielle, y compris celle de Jean-Pierre Bemba, jugée «irrecevable», à la suite de ses démêlés avec la CPI ${ }^{106}$ qui l'avait autrefois condamné à un an de prison et au paiement de 300000 euros pour subornation ${ }^{107}$ des témoins. Nombreux Congolais ont pris « subornation» pour « subordination».

92. Les « invalidés » font recours à la Cour constitutionnelle-un véritable filtre institutionnel- qui réhabilite trois candidats et confirme l'invalidation de la candidature d'Adolphe Muzito (ancien Premier ministre de JKK); celle du nonagénaire Antoine Gizenga (lui aussi ex-Premier ministre de JKK) et de Jean-Pierre Bemba, ex-Vice-Président de la RDC, ex-collaborateur de JKK et ex-détenu de la CPI- qui voient se volatiliser leur rêve à la présidentielle de déc.2018 que Fatshi remportera haut la main, moyennant quelques arrangements certains avec JKK. Tout pouvoir vient de Dieu. Peut-être!

93. Des bruits avaient pourtant couru au sujet d'une éventuelle « invalidation » de la candidature de Fatshi pour «faux diplômes» présentés à la CENI. Cette rumeur fut vite étouffée dans l'œuf et y revenir actuellement ne serait qu'un débat stérile, alimenté par ceux-là qui veulent réellement chercher des poux dans la tête de Fatshi.

94. La candidature d'Emmanuel Ramazani Ramazani Shadary à la présidentielle sonne comme un ouf soulagement pour les anti-glissement qui pourtant ne décolèrent pas, suspectant JKK de jouer au scenario «Poutine-Medvedev ${ }^{108}$.

95. Le dauphin de JKK a «glorieusement » échoué à l'élection présidentielle du 30 déc.2018. Cependant, les détracteurs de JKK suspectent ce dernier de nourrir les ambitions de revenir au pouvoir, au terme du quinquennat de Fatshi qui, selon la CENI et la Cour Constitutionnelle, l'a remporté, haut la main, contre Martin Fayulu Madidi et Emmanuel Ramazani Shadari, à l'issue de cette parade électorale!

96. Samy Badibanga, cadre dissident de l'UDPS, ancien Premier Ministre de JKK, s'interroge sur l'avenir politique de ce dernier : "Prépare-t-il un comeback pour les élections de 2023 ? A cette date, Corneille Nangaa ne sera plus à la tête de la CENI (...) pour arranger les chiffres. Qui voudra alors voter Kabila? ${ }^{109}$.

97. A3 de répliquer : «Je ne sais pas s'il vise 2023, mais, d'ici-là, il ne veut pas se créer de handicap ${ }^{110}$.

98. Le 12 sept.2018, réunis à Bruxelles, les partis politiques d'opposition peaufinent leurs stratégies.

\footnotetext{
${ }^{103} \mathrm{https} / / / \mathrm{www}$.jeuneafrique.com/mag/614022/politique/rdc-andre-alain-atundu-mp-les-elections-ne-sont-pas-la-fin-du-monde/ (1er aout19).

104 J.D.MALLET, La tragédie et la comédie, Ed. Hatier, Saint-Amand, 2001.p.69.

${ }^{105}$ Agence Nationale des Renseignements.

${ }^{106}$ Cours Pénale Internationale.

${ }^{107}$ Subornation : dérivé de "suborner": monnayer, corrompre, déterminer ou influencer (une personne, un témoin) à déposer en justice d'une façon contraire à la vérité. Cela n'a rien à voir avec la subordination qui renvoie à la soumission, à l'assujettissement, à la dépendance, etc.

${ }^{108}$ Le Président Vladimir Poutine a cédé le fauteuil présidentiel à Medvedev Dimitri en 2008, devenant ainsi le premier ministre de ce dernier pour revenir et se faire élire président en 2012, jusqu'à ce jour !

${ }^{109}$ Jeuneafrique $\mathrm{n}^{\circ} 3034$ du 3 au 9 mars 2019, p.29.

${ }^{110}$ Ibid.
} 
99. Le 11 nov.2018, rencontre stratégique de Genève, consacrant la candidature unique de Martin Fayulu à la tête de la coalition Lamuka, grâce aux bons offices de la Fondation Koffi Annan. Probablement avec l'appui financier de l'Union Européenne, de Washington et de l'ONU.

100. Le 23 nov.2018, retirant leurs signatures de l'accord de Genève, deux opposants de taille, Vital Kamerhe de l'Union pour la Nation Congolaise (UNC) et Fatshi (UDPS), se réunissent à l'Hôtel Serena de Nairobi, sous les auspices de l'Ambassade des Etats-Unis d'Amérique au Kenya, pour contrecarrer le conclave de Genève, visiblement soutenu par les Européens. Des assises de Nairobi naît une autre coalition dite «Cap pour le Changement» $(\mathrm{CACH})$ qui présente Fatshi à la présidentielle du 30 déc.2018. Vital Kamerhe, véritable animal politique, est maître à bord !

101. Le 8 Oct.2018:Fatshi est reçu en audience privée par le Président ougandais Yoweri Kaguta Museveni à Kampla. Jusque-là, personne ne sait exactement de quoi ils ont parlé.

102. Le 18 Oct.2018 JKK reçoit à Kinshasa-suite du premier round diplomatique précité- un émissaire du Président Yoweri Kaguta Museveni, Monsieur Sam Kahamba Kutesa, Ministre ougandais des Affaires étrangères ${ }^{111}$. Rien de substantiel et de véridique n'a filtré de ces deux rencontres stratégiques. Mais on sent que quelque chose se tramait déjà en coulisses.

\section{III.9. Cap vers les élections : règlement des comptes, black-out médiatique, tractations de dernière minute et passation civilisée du pouvoir}

103. Le 13 déc.2018, aux petites heures du matin, un incendie ravage l'entrepôt de la CENI à Kinshasa. Les opposants accusent le régime de JKK d'avoir mis le feu sur cet entrepôt afin de retarder ou reporter les élections ${ }^{112} \ldots$ aux calendes grecques ! Le régime de JKK proteste et dément.

104. En représailles au refus de $l^{\prime} U E^{113}$ de lever les mesures de sanction contre les dignitaires du régime JKK, Kinshasa déclare M. Bart Ouvry, Ambassadeur de l'UE en RDC, «Persona non grata », le 27 déc. 2018.

105. Le diplomate européen a 48 heures pour quitter le territoire congolais. L'UE déplore cette expulsion, la jugeant "totalement injustifiée" « à la veille d'élections très difficiles en RDC ».

106. Alors que les élections générales sont à deux doigts, les règlements de comptes continuent entre les alliés d'hier et divorcés d'aujourd'hui.

107. Le 28 déc.2018: «Joseph Kabila traite Moïse Katumbi de Judas Iscariote »" Selon JKK, « Katumbi me rappelle Judas, qui était parti avec beaucoup d'argent et s'est pendu de lui-même. Il a fait son choix. A lui d'assumer $\gg .{ }^{115}$

108. Coup sur coup, Moïse Katumbi réplique : «Ce n'est surtout pas à Joseph Kabila de me traiter de Judas. Je crois qu'il doit lire la Bible. Aujourd'hui, Monsieur Kabila doit faire son examen de conscience. Il est resté au pouvoir de 2016 à 2018, il a trahi la Constitution congolaise, il a trahi le peuple congolais, il a tué à l'est du Congo, à Béni et dans les deux Kasaï, il y a l'insécurité qui règne aujourd'hui ». Il se justifie : «Moi, je ne voulais pas rester avec l'incarnation du mal, c'est pourquoi j'ai quitté Kabila. Si lui peut se comparer au Christ, je demande à la population congolaise de nous juger. Pour moi, le Judas c'est Kabila ${ }^{116}{ }^{116}$

\footnotetext{
${ }^{111}$ Jeuneafrique $\mathrm{n}^{\circ} 3034$ du 3 au 9 mars 2019.

${ }^{112}$ https://afrique.lalibre.be/29595/rdc-incendie-de-lentrepot-de-la-CENI-le-temoignage-qui-accuse-le-pouvoir/ (11sept19).

${ }^{113}$ Union Européenne.

${ }^{114} \mathrm{http}$ ://www.kribiosuniversal.com/post/reglement-de-comptes-kabila-traite-katumbi-de-judas-iscariote- (11sept19).

${ }^{115}$ http://kribiosuniversal.com/post/reglement-de-comptes-kabila-traite-katumbi-de-judas-iscariote- (26mars2021)
}

${ }^{116} \mathrm{https} / / / 7$ sur7.cd/m-katumbi-ce-nest-pas-surtout-a-joseph-kabila-de-me-traiter-de-judas-je-crois-quil-doit-lire-(23mars2021) 
Dans cette rhétorique de la polémique, on note le recours de Moïse Katumbi à deux types d'armes verbales dont (i) la retorsio argumenti et (ii) la refutatio ad auditores où, respectivement, (i) l'accusé retourne l'argument contre l'accusateur tout en (ii) se tournant vers l'auditoire/ lectorat dans le but de provoquer la pitié, le rire, l'indignation ${ }^{117}$.

109. Comme en 2006 pour Joseph Kabila, Vital Kamerhe a abattu un travail de bénédictin en faveur de Fatshi. Bien des Congolais voient en lui un technocrate chevronné, un animal politique, un « faiseur de rois ». Quelques indiscrétions de son fief électoral du Bushi ont sournoisement lâché, dans un proverbe de chez eux : «Oshenyer' ecirema, yena cirhwâza ${ }^{118}$.

110. Fatshi se rapproche de JKK et le 10 jan.2019, il est déclaré-contre toute attente- par la CENI, gagnant de l'élection présidentielle.

111. Le 20 janv. 2019, Fatshi est confirmé et proclamé Président-élu-de la République Démocratique du Congo par la Cour Constitutionnelle. Il tire donc son épingle du jeu, bien qu'il s'agisse d'un énième débauchage par JKK d'un membre de l'UDPS. Sur les ondes de la RFI, Alphonse Mahindo- un éminent politologue et Professeur des universités estime que, cette fois-ci, Joseph Kabila a « décapité » l'UDPS qui, par ce fait même, cesse d'exister en tant que parti politique de l'opposition ${ }^{119}$.

112. La victoire de Fatshi a soulevé des controverses tant à l'intérieur qu'à l'extérieur du pays, au sujet de la «vérité des urnes ». En effet, nombreux rapports d'observateurs, dont ceux de la CENCO, affirment et confirment par ailleurs la victoire de Martin Fayulu sur Fatshi ; et cette opinion est relayée par la fameuseet nébuleuse-Communauté internationale (UE \& Etats-Unis) et l'UA.

113. Paul Kagame du Rwanda est président en exercice de l'UA. Il aurait pu peser de tout son poids pour la restauration de la vérité des urnes tant revendiquée par Lamuka et, peut-être même, donner un coup de pouce à Martin Fayulu pour accéder au pouvoir, n'eût été la fermeté de JKK de rester le « Maestro »du processus de passation du pouvoir.

114. Cependant, le 19 janv.2019, une délégation de Kinshasa, conduite par Kalev Mutond (comprenant She Okitundu et le Général Delphin Kahimbi) arrive à Kigali "pour amadouer le Rwandais Paul Kagame très remonté contre l'annonce des premiers résultats des élections congolaises ${ }^{120}$.

115. Au lendemain des élections, les Congolais se réveillent privés de moyens de communication, principalement la connexion Internet et la messagerie téléphonique. Les réseaux sociaux sont perçus comme des armes par lesquelles les anti-glissement véhiculent des messages de contestation et relayent les appels à manifester. La coupure du réseau Internet et du signal de la RFI en RDC- par le kabiliste impénitent Lamber Mende Omalanga- fait partie des pratiques gouvernementales afin de « mieux assurer l'ordre public », avant, pendant et après les manifestations populaires, organisées contre le régime.

116. Le Figaro écrit: "Petit à petit, le pays s'enfonce dans un huis-clos. Le signal de RFI a été coupé sur la quasi-totalité du territoire et les autorités ont retiré l'accréditation de son envoyée spéciale à Kinshasa, Florence Morice ».

117. La Communauté internationale menace, dans un communiqué conjointement signé par les représentants du Canada, de la Suisse, de la France, de la Belgique, du Royaume-Unis, des Pays-Bas et de la Suède, l'UE : «Nous demandons au gouvernement de ne pas bloquer les moyens de communication et notamment l'accès à Internet ainsi que les médias ». Un vœu pieu, pendant que la fameuse Communauté internationale ne lève pas son petit doigt...

\footnotetext{
${ }^{117}$ MUTUNDA Mwembo, Op.Cit.p.102.

${ }^{118}$ Lorsque tu as décidé de venir en aide à une personne que tu aimes et qui a des lacunes ou insuffisances, il faut aller jusqu'au bout.

${ }^{119}$ http://www.rfi.fr/emission/20190124-rdc-transition-historique-investiture-tshisekedi-cohabitation-kabila (Prof. Alphonse Mahindo).

${ }^{120}$ Jeuneafrique $\mathrm{n}^{\circ} 3034$ du 3 au 9 mars 2019.
} 
118. Mike Hammer, dit «Nzita», Ambassadeur des Etats-Unis en RDC, twitte, le $1^{\text {er }}$ janvier 2019 : Je souhaite une bonne année et meilleurs væux à mes amis Congolais! Que 2019 vous apporte la paix, une transition démocratique et l'internet débloqué!».

119. Des tractations sont menées par JKK pour une passation «civilisée » du pouvoir et le Secrétaire Général de l'UDPS, Jean-Marc Kabund-plus militant qu'homme politique - a suggéré « une rencontre » entre MM. Tshisekedi et Kabila «pour préparer la passation pacifique et civilisée du pouvoir », et ce, avant même la proclamation des résultats ${ }^{121}$.

120. Cette entente secrète est perceptible à travers ces propos de Lambert Mende : «Nous n'allons pas rejeter la main tendue parce qu'il y a un temps pour tout. Un temps pour s'opposer et se disputer l'électorat, mais aussi un temps pour s'unir $\gg .{ }^{122}$.Discours digne d'un élu démagogue et opportuniste ...au détriment du peuple congolais qu'il prétend représenter.

121. Ce « deal » est vite dénoncé par Eva Bazaiba, Secrétaire Générale du MLC ${ }^{123}$ et porte-parole de Martin Fayulu: "Kabila n'a pas à dire : je veux telle personne, je ne veux pas de telle. Ce n'est pas sa résidence privée ni une affaire de famille, c'est une affaire d'État ».

\section{IV.Apotheose}

Toujours est-il qu'au lendemain du scrutin, et face au mauvais score réalisé par son dauphin, Emmanuel Ramazani Shadary, JKK est en quête d'un plan B et cherche presque désespérément avec qui négocier-afin de lui passer les rênes du pouvoir et sauver sa peau. Aussi apprend-on que "Par l'intermédiaire de Leila Zerrougui, la représentante spéciale du Secrétaire général de l'ONU à Kinshasa, il tente de sonder Martin Fayulu, le candidat donné gagnant par les observateurs de la puissante CENCO. Le champion de la plateforme Lamuka ne donne pas suite ${ }^{124}$. Parallèlement, Joseph Kabila tend une perche à l'autre candidat de l'opposition, Felix Tshisekedi, beaucoup plus ouvert à la discussion. Le 24 janvier, Kabila lui cède le pouvoir ${ }^{125}$. Et ce fut le jour mémorable de la passation dite «civilisée »-mais pas nécessairement démocratique- du pouvoir, jour historique d'une transition pacifique et apaisée en RDC, entre un président sortant et un président entrant.

122. JKK remet à Fatshi les symboles du pouvoir, ou plutôt le pouvoir symbolique, car d'autres analystes sont d'avis qu'il lui a plutôt cédé la corde, tout en gardant la chèvre ! Et, de la corde à la chèvre, il y a un fossé que Fatshi se doit de combler et de franchir! Depuis lors, la vérité des urnes est devenue un débat purement académique. Entre vérité éristique et vérité pragmatique, socialement construite, les Congolais et JKK ont fait leur choix, auquel même la Communauté internationale s'est finalement pliée, afin de ne pas jeter le bébé et l'eau du bain. Quelques mois après l'investiture du nouveau président, un diplomate de la Communauté internationale avait lâché, au sujet du nouveau régime congolais : "Nous sommes conscients que nous avons bâti sur le mensonge, mais nous n'y pouvons plus rien. Maintenant il va falloir aider FélixAntoine Tshisekedi à devenir réellement Président! ». Fructueux quinquennat au $5^{\mathrm{e}}$ Président de la RDC, et heureux comme Fatshi qui a fait un très bon voyage!

\section{Resultats \& Discussion}

Les récents affrontements observés sur la scène politique congolaise dans la phase pré-électorale d'avant déc. 2018 ont mis en lumière, d'une part, ce conflit presqu'inévitable entre légitimité et légalité, et d'autre

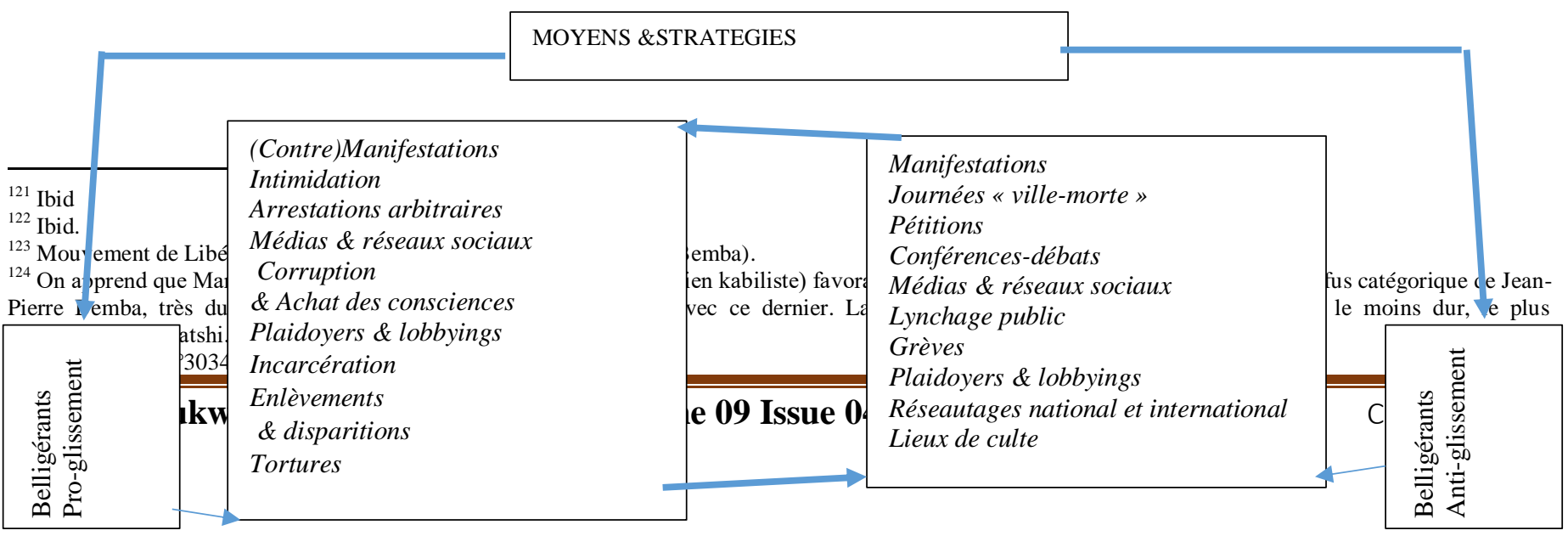


part, les mobiles qui incitent les acteurs politiques congolais à se disputer le pouvoir. La légitimité et la légalité ne préviennent pas le pouvoir en place contre toutes les formes imaginables de contestation, de

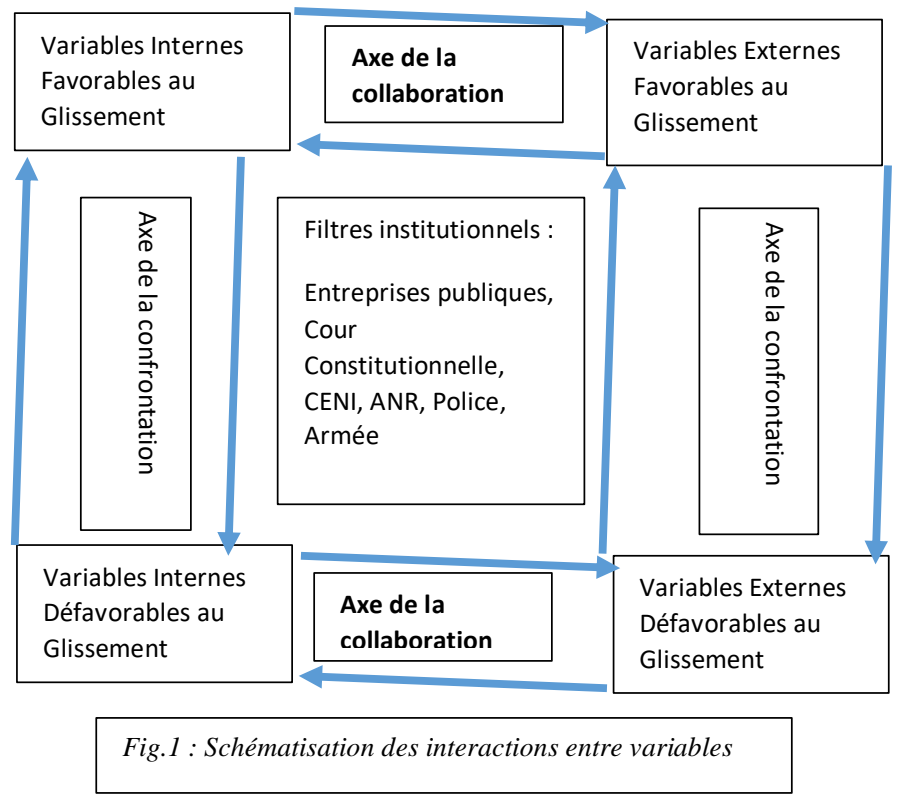

protestation et de boycottage. La première, bien que liée à la loi, ne s'apprécie pas en termes de droits en vigueur mais plutôt en termes d'idées, de représentation qu'un peuple se fait du droit à un moment déterminé. D’où son corollaire de légalité, qui est lié à l'opinion. Aussi arrive-t-il qu'on puisse reprocher aux autorités légalement investies leur manque de légitimité dès qu'elles ont manifestement perdu le soutien de l'opinion. ${ }^{126}$ Prenant appui sur quelques clauses de la Constitution, Joseph Kabila et ses lieutenants ont tenté de se maintenir au pouvoir contre la volonté collective qui, elle aussi, prenant la Constitution à témoin, a fini par forcer ces deniers à lâcher prise. L'univers politique regorge d'avantages très souvent à l'origine des affrontements, dès lors que ceux qui détiennent le pouvoir cherchent à tout prix à le conserver, même audelà du mandat constitutionnel, pendant que leurs challengers cherchent à le récupérer afin d'accéder, eux aussi, et à leur tour, aux mêmes avantages. Au menu de ces avantages qu'il peut procurer à ceux qui le détiennent et l'exercent, le pouvoir politique ou le pouvoir d'Etat donne un accès facile au prestige, à l'honneur, à la puissance, à l'argent, aux « femmes ${ }^{127}$. Les pro- et anti- glissement ont dû faire recours à leurs alliés, internes et externes, afin de parvenir à leurs fins. Les discours, à eux seuls, n'ont pas suffi et les actes ont été posés par les uns et les autres afin de faire prévaloir leurs arguments. Les variables internes et externes défavorables au glissement, engagés dans l'axe de la collaboration ont fini par faire fléchir les variables internes et externes favorables au glissement, engagées dans l'axe de la collaboration et qui avaient pourtant à leur disposition, les filtres institutionnels pour maintenir le statu quo. Les pro et anti-glissement internes, engagés dans l'axe de la confrontation, ont misé sur l'appui de leurs alliés respectifs, engagés, eux aussi, dans l'axe de la confrontation.

Les «belligérants "pro- et anti-glissement recourent souvent à des moyens stratégiques identiques dont la manifestation et contre-manifestation, les médias et réseaux sociaux, bien que ces derniers aient été considérés comme l' «arme » la plus accessible et manipulable pour les appels à manifester. En dépit des forces répressives dont disposait le régime de JKK, le recours au black-out médiatique et d'Internet, les

\footnotetext{
${ }^{126}$ B.N. LUMANU-MULENDA, Communication politique FCK, Kinshasa, 1999-2000,p.9 (notes de cours) (inédit).

${ }^{127}$ B.N. LUMANU-MULENDA, Communication politique...p.3. Ici, Lumanu-Mulenda réifie les femmes et les assimile aux avantages matériels et autres délices que procure le pouvoir politique aux politiciens en RDC, en Afrique et ailleurs. Cette mentalité date de l'époque de feu le Président Mobutu et semble avoir traversé d'un régime à l'autre.
} 
anti-glissement n'ont pas fléchi. Les pro-glissement externes été largement évoqués dans la première partie de cette étude publiée ailleurs. Quant aux internes, y compris les «délinquants » constitutionnels qui tentaient de «brader » la constitution en vue d'un $3^{\mathrm{e}}$ mandat, ils ont enfin lâché le régime de Joseph Kabila et l'intéressé, occasionnant ainsi un déséquilibre des forces dont le point d'orgue fut l'alternance politique tant revendiquée.

\section{Conclusion}

Les résultats de cette étude attestent que les belligérants récemment observés sur la scène politique congolaise n'ont pas été les seuls et réels acteurs à en avoir déterminé, les uns l'épuisement et l'effondrement du régime, et les autres, les efforts d'autoconservation. Nombreux acteurs ont agi dans l'ombre, tant de l'intérieur que de l'extérieur. Il est fort à croire que l'ingérence extérieure dans les affaires intérieures de l'Etat congolais et la diplomatie de couloir, menée par une diversité d'acteurs même anonymes, ont apporté de l'eau au moulin de l'idéologie contestataire du statu quo pour faire fléchir le régime de Joseph Kabila dont la légitimité et la légalité devenaient de plus en plus contestée. Des gouttes d'eau à la fois internes et externes ont fini par faire déborder le vase. Le régime kabiliste, excellant en maladresses, fut pressé de toutes parts et s'est émoussé et effondré, tout en gardant une large marge de manœuvre afin de survivre à lui-même et s'auto-conserver. On en saura davantage les mois et années à venir.

D'un côté, les personnages-clés favorables (internes et externes) au glissement sont notamment JKK, Lambert Mende, She Okitundu, CENI, Cour Constitutionnelle, ANR, Police \& Armée, Evariste Boshab, Jean Cyrus Mirindi, André-Alain Atundu (A3). De l'autre, les contestataires du statu quo, internes et externes, sont principalement les Mouvements citoyens, la population représentée par une diversité de manifestants, la CENCO, Laurent Cardinal Monsengwo, Mgr François-David Ekofo, les chefs de file des partis politiques dits d'opposition qui espèrent récupérer les initiatives des Mouvements citoyens, les médias, les activistes des droits humains, le Vatican, les représentations diplomatiques, etc. Le mutisme de la plupart de Chefs d'Etats africains, notamment ceux de la CEPGL, de la CIRGL et de la SADC ${ }^{128}$ sur le sort politique de leur pair JKK laisse croire qu'ils ont été contraints à se plier à la volonté de la Communauté internationale, pour ne pas s'attirer la foudre de celle-ci.

\section{Remerciements}

Cette étude a bénéficié des apports médias dont les écrits en ont constitué le corpus. Les discours, les faits et gestes des «belligérants» pro- et anti-glissement rapportés dans cette étude demeurent une mine d'information collectée par des acteurs engagés quant à la crise politique congolaise à divers niveaux, national et international. Les témoignages de bien d'informateurs de qualité, bien que « hors-médias », ont enrichi la discussion de cette étude. Notre gratitude à tous ces acteurs et informateurs, même aux plus discrets et anonymes !

${ }^{128}$ Communauté de Développement de l'Afrique Australe. 